\title{
PLANEJAMENTO ESTRATÉGICO PARA UM CENTRO DE FORMAÇÃO DE CONDUTORES EM UM MUNICÍPIO DE PEQUENO PORTE DO NOROESTE DO RS
}

Danieli Alini WELTER ${ }^{1}$

Kauana Patrícia MALLMANN ${ }^{2}$

Mauro Alberto NÜSKE ${ }^{3}$

Marcos Paulo Dhein GRIEBELER ${ }^{4}$

\author{
${ }^{1}$ Bacharel em Administração (SETREM), email: danialini@ hotmail.com \\ ${ }^{2}$ Bacharel em Administração (SETREM), email: kauana_patricia@hotmail.com \\ ${ }^{3}$ Administrador e Especialista em Administração Financeira (SETREM), Mestre em Engenharia de Produção \\ (UFSM), Doutorando em Desenvolvimento Regional (UNIJUÍ), e-mail: mauronuske@gmail.com \\ ${ }^{4}$ Professor da Universidade Regional do Noroeste do RS (UNIJUÍ), Doutor em Desenvolvimento Regional pela \\ Universidade de Santa Cruz do Sul (UNISC), e-mail: marcos.dhein@unijui.edu.br
}

Recebido em: 06/07/2016 - Aprovado em: 05/01/2017 - Disponibilizado em: 01/07/2017

\begin{abstract}
RESUMO
O presente trabalho de conclusão de curso tem por objetivo desenvolver uma proposta de planejamento estratégico para um Centro de Formação de Condutores - CFC de um município de pequeno porte da Região Noroeste do Rio Grande do Sul. O problema de pesquisa questiona, quais aspectos são relevantes para a definição de objetivos estratégicos para um CFC? Utilizaram-se os métodos de abordagem dedutivo, qualitativo e quantitativo com o procedimento da pesquisa descritiva e o estudo de caso. Para a coleta de dados e sua análise, foram utilizados, respectivamente, a observação, a entrevista e o questionário realizados com o diretor da empresa. Buscou-se conceitos de autores para abordar assuntos referentes ao trabalho, como administração e gestão estratégica, planejamento estratégico, conceitos dos propósitos da organização, sobre análise de ambiente interno e externo, postura estratégica, objetivos estratégicos, planos de ação e ferramentas de controle do planejamento estratégico. Em relação ao problema da pesquisa, pôde-se constatar que é de grande importância à empresa ter definido os seus propósitos. Foi fundamental desenvolver a análise de ambiente interno e externo, pois a partir desta foi possível elaborar os objetivos estratégicos bem como os planos de ações. Através da análise de ambiente verificou-se em qual dos quadrantes da postura estratégica a empresa se encontra, sendo o desenvolvimento a postura recomendada.
\end{abstract}

Palavras-chave: Planejamento Estratégico. Objetivos Estratégicos. Postura Estratégica. Planos de Ação. Desenvolvimento.

\begin{abstract}
The project has as objective to develop a strategic plan for Driver's License training in a small city in Northwest Region of RS. The research seeks to identify which aspects are relevant in the definition of strategic objectives for a Driver's License training. It was used deductive, qualitative and quantitative methods together with descriptive and a case study. For data collection and analysis, were used, respectively, observation, interview and a questionnaire conducted with the director of the company. Researching authors concepts to address subjects related to this study, such as administration, strategic management and planning, concepts of the purposes of the organization, else on analysis of internal and external environment, strategic posture, strategic objectives, action plans and strategic planning tools of control. Regarding the research, we could see that it is of very important to the company have set their purposes. It was essential to develop the analysis of internal and external environment, because from this it was possible to develop strategic objectives and action plans. Through the analysis of the environment it was found which quadrants of the strategic posture the company is located, and later the company was being developed and targeted to the recommended position.
\end{abstract}

Keywords: Strategic Planning. Strategic Objectives. Strategic Posture; Action Plans; Development. 


\section{INTRODUÇÃO}

$\mathrm{O}$ atual modelo econômico e os processos de globalização causam a sensação de que o tempo está passando cada vez mais rápido. Tem-se a cada dia e a todo momento uma quantidade avassaladora de informações. A internet permite ter acesso a tudo que possa interessare, todo este conteúdo, irá influenciar a vida das pessoas de alguma maneira; além disso, a capacidade de raciocínioestá sendo vencida pela velocidade e pela quantidade de informações existentes.

Essa aceleração do ritmo de vida das pessoas e das empresas, direcionapara a tomada de decisões imediatas, sem muita análise e geralmente com resultados a curto prazo, isto é, as tarefas de refletir e planejar tornam-se cada vez mais difíceis de acontecer.

Essa nova realidade exige empresas bem preparadas, uma organização com objetivos e metas traçadas, conhecimento do mercado em que está atuando, análise do ambiente e que esteja preparada para as surpresas que esse possa oferecer, antecipando-se aos acontecimentos futuros.

A ferramenta utilizada para o controle deste processoé o planejamento estratégico, que visa proporcionar um desenvolvimento a curto ou longo prazo com o objetivo de as metas serem alcançadas de forma eficiente. Com o planejamento estratégico, além das decisões serem mais eficazes, os recursos e os esforços das empresas são concentrados nas ações que devem ser executadas.
O planejamento estratégico é uma das ferramentas que possibilita aos gestores administrar melhor os seus negócios e, independentemente do tamanho da empresa, é necessário estabelecer metas, parâmetros e estratégias, facilitando a gestão da organização, tornando-se essencial para qualquer tomada de decisão e para sua execução.

A partir deste contexto, este estudo trabalho propôs a realização de um planejamento estratégico para um Centro de Formação de Condutores, procurando estabelecer quais os aspectos que são relevantes para a definição de objetivos estratégicos da empresa.

\section{ASPECTOS METODOLÓGICOS}

Para o estudo utilizou-se o método dedutivo, buscando teorias em autores e trabalhos científicos para que fosse possível elaborar e dar sustentação ao referencial teórico bem como para orientar a construção de um planejamento estratégico para um CFC.

O método qualitativo foi utilizado para a compreensão detalhada do funcionamento e da estrutura da organização, além de entrar em contato direto com o ambiente e a situação que está sendo investigada permitindo um contato com gestores e colaboradores, descrevendo e detalhando o significado de características apresentadas durante a entrevista, em lugar da produção de medidas quantitativas.

4 
Já o método quantitativo foi utilizado na análise de ambiente, a partir da pontuação atribuída a cada um dos aspectos avaliados, tanto no ambiente interno como no externo, bem como na postura estratégica elaborada.

Por meio da aplicação da análise de ambiente interno e externo, foi utilizada a pesquisa descritiva com base na visão do gestor, para relatar informações de caráter financeiro, tecnológico, comercial e de recursos humanos, por meio da análise ambiental.

O estudo de caso foi utilizado para uma análise aprofundada dos procedimentos da empresa, proporcionando maior clareza das atividades desenvolvidas e da realidade da empresa.

A técnica de coleta de dados foi realizada através de questionário, aplicado através de entrevista ao diretor da empresa, contendo questões relacionadas ao ambiente externo, onde foram apontadas oportunidades e ameaças e com relação ao ambiente interno, pontuados pontos fortes e fracos.

A técnica da observação foi empregada a fim de coletar dados e extrair informações relevantes ao estudo, na forma de visitas e conversas informais com os envolvidos da organização.

$\mathrm{Na}$ técnica de análise de dados foi realizada a extração de todos os dados para posterior análise, obtenção de resultados e entendimento dos mesmos.

\section{REVISÃO BIBLIOGRÁFICA}

ADMINISTRAÇÃO ESTRATÉGICA

Conforme Matias-Pereira (2011), o processo da administração estratégica, tornase contínuo, pois a interação decorre do processo cíclico da administração estratégica, inicia-se na primeira etapa, vai até a última e retorna à primeira. A interação com o ambiente surge para assegurar os resultados esperados, uma vez que as transformações levam as empresas a se adaptarem.

Para Ansoff (1993) apud MatiasPereira (2011), a administração estratégica é uma abordagem sistêmica de gestão de mudanças estratégicas e é uma responsabilidade importante e cada vez mais essencial da administração geral: posicionar e relacionar a organização ao seu ambiente, assegurando resultados continuados e antecipando eventuais surpresas. Pode-se resumir que a administração visa manter uma organização como um conjunto integrado ao seu ambiente, num processo evolutivo, contínuo e interativo.

\section{PLANEJAMENTO ESTRATÉGICO}

Segundo Chiavenato e Sapiro (2003), planejamento estratégico é o processo contínuo de tomar decisões atuais que envolvem riscos, organizar sistematicamente as atividades necessárias à execução dessas decisões e medir o resultado dessas decisões em confronto com as expectativas alimentadas. 
Para Matias-Pereira (2011), planejar estrategicamente é utilizar técnicas administrativas para ordenar ideias de forma que se possa criar uma visão do caminho a ser seguido. Ordenando as ideias, é necessário ordenar as ações, implementando assim o plano estratégico para que a organização caminhe de acordo com o que é planejado.

Conforme Kich e Pereira (2011) planejamento estratégico é um processo que consiste na análise dos pontos fortes e fracos da organização e das oportunidades e ameaças do ambiente externo, com o objetivo de formular estratégias e ações estratégicas com o intuito de aumentar a competitividade.

\section{NEGÓCIO, VISÃO, MISSÃO, PRINCÍPIOS} E VALORES DA EMPRESA

Para Chiavenato e Sapiro (2003), é preciso entender o produto ou serviço que o cliente esta comprando e evitar a armadilha de olhar apenas para o produto físico oferecido. O negócio pode ser definido nas seguintes dimensões: grupos de clientes, necessidades de clientes e tecnologia.

Segundo Oliveira (2013), na visão identifica-se quais são as expectativas e os desejos dos acionistas, conselheiros e elementos da alta administração da empresa, tendo em vista que esses aspectos proporcionam o grande delineamento do planejamento estratégico a ser desenvolvido e implementado. Já a missão é a razão de ser da empresa. Nesse ponto procura-se determinar qual o negócio da empresa, por que ela existe, ou ainda quais os tipos de atividades que a empresa deverá concentrar-se no futuro.

Para Sampaio (1999), os princípios ou valores da empresa são aquilo que ela acredita que servirão como orientadores para o processo decisório e para o comportamento da empresa no cumprimento da sua missão.

\section{ANÁLISE DO AMBIENTE}

Análise do ambiente, para Vasconcelos Filho e Pagnoncelli (2001), é um conjunto de técnicas que permitem identificar e monitorar permanentemente as variáveis competitivas que afetam a performance da empresa.

Andrade (2012) acredita que a análise do ambiente externo tem por finalidade buscar o máximo de informações possíveis a respeito das condições ambientais externas, que constituem o cenário no qual a organização esta inserido e o relacionamento entre ambos.

Para Sampaio (1999), as ameaças são situações externas que colocam a empresa diante de dificuldades para atingir os objetivos ou a perda de mercado e ainda a redução da rentabilidade.

As oportunidades, para Vasconcelos Filho e Pagnoncelli (2001), são situações externas atuais ou futuras que, se adequadamente aproveitadas pela empresa, podem influenciá-la positivamente.

Segundo Andrade (2012) a análise interna é necessária para adaptar-se às 6 
condições do entorno. O estoque de recursos, a habilidade dos empregados, a estrutura da empresa, a cultura e o clima organizacional, além de outros fatores internos, é que irão determinar as suas possibilidades de adaptação e, consequentemente, o êxito de suas estratégias.

Sampaio (1999) identifica os pontos fortes como sendo características internas da empresa, tangíveis ou não, que fornecem uma vantagem competitiva sobre os seus concorrentes ou uma facilidade para atingir seus objetivos.

Para Andrade (2012), um ponto fraco é quando a empresa não tem algo, ou não faz muito bem, comparado com as outras, ou uma condição que a coloque em desvantagem. $\mathrm{O}$ ponto fraco não torna necessariamente a empresa vulnerável competitivamente, dependendo de quanto ele pese no mercado.

Segundo Matias-Pereira (2011), a matriz SWOT é composta por quatro palavras inglesas strenghts (forças); weaknesses (fraquezas); opportunities (oportunidades) e threats (ameaças), que geram quatro matrizes: a postura de sobrevivência, manutenção, crescimento e desenvolvimento. A análise SWOT tem como objetivo definir estratégias para manter pontos fortes, reduzir a intensidade de pontos fracos, aproveitando as oportunidades e protegendo-se das ameaças.

\section{OBJETIVOS ESTRATÉGICOS}

Conforme Chiavenato e Sapiro (2003), os objetivos são estabelecidos a partir dos anseios e expectativas humanas em relação a uma condição futura ideal.

Para Maximiano (2000), os objetivos são a concretização dos resultados que a empresa pretende realizar. É por meio dos objetivos que a empresa tem o desafio de construir o seu futuro. Para que seja possível atingi-los, os mesmos devem dar as condições necessárias; devem ter prazo definido, ser mensuráveis, coerentes, e não deixar de serem desafiadores.

\section{PLANOS DE AÇÃO}

Com o desenvolvimento do planejamento estratégico a empresa estará adotando um novo estilo de gestão. Para Andrade (2012), o planejamento estratégico lida com questões de caráter geral, de abrangência ampla e voltada ao longo prazo. Os planos de ação lidam com questões táticas e operacionais, voltadas para o médio e curto prazo e moldam as estratégias em planos mais concretos e de fácil entendimento para todos os setores da empresa.

Para a elaboração de um plano de ação, é necessário ter em mente as causas do problema e realizar cada etapa de maneira cuidadosa, sempre de forma correta sendo que as perguntas da Figura 1 devem ser todas respondidas. 


\begin{tabular}{|c|c|c|c|c|c|}
\hline \multicolumn{5}{|c|}{ Plano de ação } \\
\hline O que? & Quem? & Quando? & Onde? & Por que? & Como? \\
What? & Who? & When? & Where? & Why? & How? \\
\hline
\end{tabular}

Figura 1 - Modelo Plano de Ação

Fonte: Costa (2007)

Para Costa (2007), cada objetivo e cada meta deve conter planos de ação específicos, para assegurar que as ações e os passos necessários para a implementação das estratégias combinadas sejam executadas e acompanhadas por pessoas previamente alocadas.

\section{FERRAMENTAS DE CONTROLE DO} PLANEJAMENTO ESTRATÉGICO

Todas as atividades da empresa necessitam ser monitoradas diariamente. Por meio das ferramentas de controle que visam auxiliar e orientar as empresas no processo de tomadas de decisões, consequentemente existirá, o controle de seus negócios, conquistando o que se esperava.

O processo de controle definido por Oliveira (2013) é apresentado em quatro etapas; o estabelecimento de padrões de avaliação, medida de desempenho real, comparação entre o desempenho real e o desejado e a ação corretiva. Hrebiniak (2006) apud Kich e Pereira (2011) afirma que a empresa tem de buscar a integração entre planejamento, execução e controle estratégico.

\section{APRESENTAÇÃO, DISCUSSÃO E ANÁLISE DOS RESULTADOS}

Para a definição do negócio é necessário fazer a seguinte pergunta, qual é o nosso negócio? Depois de respondida, é preciso estabelecer um espaço do setor onde a empresa atua com a finalidade de identificar oportunidades de mercado.

O negócio deve ser definido tendo em vista os benefícios e não os produtos ou serviços, portanto, o negócio da empresa ficou assim definido:

\section{"Capacitação ao dirigir."}

A capacitação está relacionada ao termo habilitação que é o principal objetivo da organização.

Dirigir está voltado em conduzir veículos automotores de forma segura e de acordo com as leis brasileiras de trânsito.

Para a elaboração da missão, é preciso ter a sensibilidade e a humildade para, mesmo tendo sucesso, perceber as mudanças e tendências do ambiente e incorporá-las na missão, sendo necessário a interpretação de algumas questões como:

- O que deve fazer a empresa?

- Para quem deve fazer?

- Para que deve fazer?

- Como deve fazer?

- Onde deve fazer?

- Qual responsabilidade social deve ter? 
A missão do CFC da empresa objeto do estudo ficou assim definida:

"Formar condutores em um centro de treinamento especializado, conscientizando-os sobre a valorização da vida capacitando-os para o diaa dia no trânsito, buscando a satisfação de todos os envolvidos."

A visão precisa ser definida de forma clara, vislumbrando o futuro desejado pela empresa e quais as aspirações desejadas.

A visão do CFC ficou assim definida:

"Ser reconhecida na região pela qualidade de serviços na formação de condutores até 2019, através de uma equipe e infraestrutura especializadas e comprometida com a valorização da vida."

Os princípios e valores de uma organização servem para controlar as decisões e os comportamentos da empresa.

A definição de princípios e valores pode ser realizada a partir de um resgate cultural da empresa. É fundamental identificar os traços marcantes do comportamento da mesma ao longo dos anos.

Os princípios e valores definidos para o CFC foram:

- Comprometimento;

- Qualidade;

- Tecnologia;

- Capacitação;

- Valorização da Vida;

- Ética;

- Respeito;

- Legalidade.
Na sequência, realizou-se a análise de ambiente, através da identificação de ameaças, oportunidades, forças e fraquezas que afetam a empresa no cumprimento de seu objetivo. Esta análise é uma técnica que permite a empresa identificar o que está acontecendo no ambiente de atuação da empresa.

A análise foi realizada através de formulário estruturado e respondido pelo diretor da empresa. No ambiente externo, foram apontadas oportunidades e ameaças, e no ambiente interno, pontos fortes e fracos, com notas de 1 a 5 e -1 a -5 , respectivamente, onde 1 e -1 são as menos relevantes, e 5 e -5 as mais relevantes.

A Figura 2 apresenta os principais itens que foram diagnosticados como oportunidades e ameaças do ambiente externo.

\begin{tabular}{|l|c|l|c|}
\hline Oportunidades & Nota & \multicolumn{1}{|c|}{ Ameaças } & Nota \\
\hline Poder de Fogo & 5 & Mídia & -4 \\
\hline $\begin{array}{l}\text { Capacidade de } \\
\text { Serviços }\end{array}$ & 5 & $\begin{array}{l}\text { Políticas de } \\
\text { Prazos }\end{array}$ & -5 \\
\hline $\begin{array}{l}\text { Estratégias de } \\
\text { Marketing }\end{array}$ & 4 & $\begin{array}{l}\text { Políticas de } \\
\text { Preços }\end{array}$ & -5 \\
\hline Mercado Alvo & 5 & Pontos Fracos & -5 \\
\hline Mídia & 4 & Educação & -4 \\
\hline $\begin{array}{l}\text { Políticas de } \\
\text { Preços }\end{array}$ & 5 & $\begin{array}{l}\text { Implicações } \\
\text { Governamentais }\end{array}$ & -4 \\
\hline Pontos Fortes & 4 & & \\
\hline $\begin{array}{l}\text { Qualidade dos } \\
\text { Bens e Serviços }\end{array}$ & 5 & & \\
\hline Tecnologia & 5 & & \\
\hline $\begin{array}{l}\text { Decisão de } \\
\text { Compras }\end{array}$ & 5 & & \\
\hline Fidelização & 4 & & \\
\hline $\begin{array}{l}\text { Qualidade de } \\
\text { matéria-prima }\end{array}$ & 4 & & \\
\hline $\begin{array}{l}\text { Saúde } \\
\text { Financeira }\end{array}$ & 4 & & \\
\hline
\end{tabular}

Figura 2 - Resumo do ambiente externo 
A Figura 3 apresenta os principais itens que foram diagnosticados como pontos fortes e fracos no ambiente interno.

\begin{tabular}{|l|c|l|c|}
\hline \multicolumn{1}{|c|}{ Pontos Fortes } & Nota & Pontos Fracos & Nota \\
\hline Inovação & 4 & Força de Vendas & -4 \\
\hline Marca & 5 & Custos & -5 \\
\hline $\begin{array}{l}\text { Relacionamento } \\
\text { com Clientes }\end{array}$ & 4 & Cobranças & -5 \\
\hline $\begin{array}{l}\text { Qualidade de } \\
\text { Equipamentos }\end{array}$ & 5 & $\begin{array}{l}\text { Políticas de } \\
\text { Investimentos }\end{array}$ & -4 \\
\hline Processo decisório & 4 & & \\
\hline Transparência & 4 & & \\
\hline $\begin{array}{l}\text { Administração de } \\
\text { Caixa }\end{array}$ & 5 & & \\
\hline Capital de Giro & 4 & & \\
\hline Controle & 4 & & \\
\hline Custos X Receitas & 5 & & \\
\hline Instalações & 4 & & \\
\hline Nível Tecnológico & 5 & & \\
\hline Produtividade & 4 & & \\
\hline
\end{tabular}

Figura 3 - Resumo do ambiente interno

Para o estabelecimento da postura estratégica da empresa são abordados três critérios: a missão da empresa; a relação existente entre as oportunidades e ameaças e a relação entre os pontos fortes ou fracos comparados com as oportunidades e ameaças do ambiente.

A análise SWOT tem o objetivo de cruzar as oportunidades e as ameaças externas da organização com seus pontos fortes e fracos.

\begin{tabular}{|c|c|c|c|c|}
\hline & \multicolumn{2}{|c|}{ Análise Interna } \\
\hline & & & \multicolumn{2}{|c|}{ Predominância de } \\
\hline & & & Pontos Fortes & Pontos Fracos \\
\hline \multirow{2}{*}{ 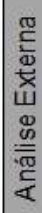 } & \multirow{2}{*}{ 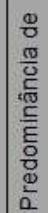 } & Oportunidades & Desenvolvimento & Crescimento \\
\hline & & Ameaças & Manutenção & Sobrevivência \\
\hline
\end{tabular}

Figura 4 - Modelo da postura estratégia da empresa Fonte: Oliveira (2013).

Na elaboração da postura estratégica seguiu-se critérios de autores, ficando definidas as corres: vermelha para correlação forte, verde para correlação média, amarelo para correlação fraca e branco quando não há correlação, como pode ser observado na Figura 5.

\begin{tabular}{|l|l|}
\hline \multicolumn{2}{|c|}{ SIMBOLOGIA } \\
\hline & Correlação Forte \\
\hline & Correlação Média \\
\hline & Correlação Fraca \\
\hline & Sem correlação \\
\hline
\end{tabular}

Figura 5 - Simbologia da postura estratégica

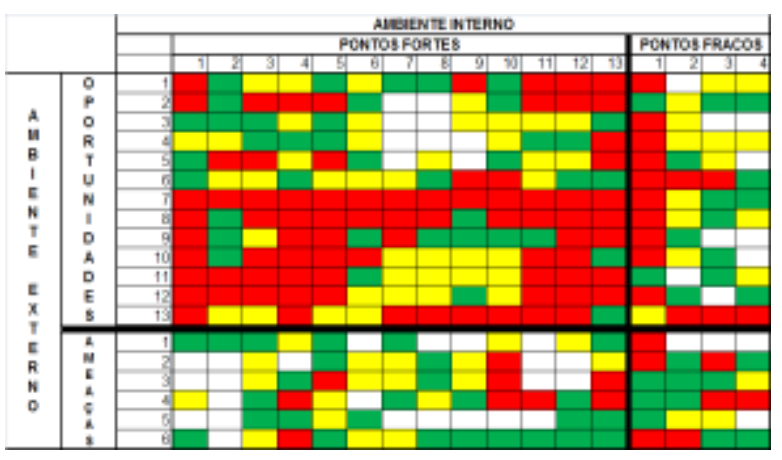

Figura 6 - Postura Estratégica da Empresa

Após a análise do ambiente interno e externo da empresa, desenvolveu-se uma avaliação da postura estratégica definindo a correlação existente entre o cruzamento das oportunidades e ameaças, pontos fortes e pontos fracos, que pode ser observado na 
Figura 6. Desta forma, pode-se identificar à qual quadrante a empresa pertence e possibilitando verificar a estratégia adequada a ser aplicada.

Desenvolveu-se um resumo da postura estratégica (Figura 7) no intuito de facilitar o entendimento. Após a análise destes dados, pode-se observar que a empresa encontra-se no quadrante de desenvolvimento, pois foi neste que ela apresentou maiores pontuações quanto às correlações forte (80) e média (39).

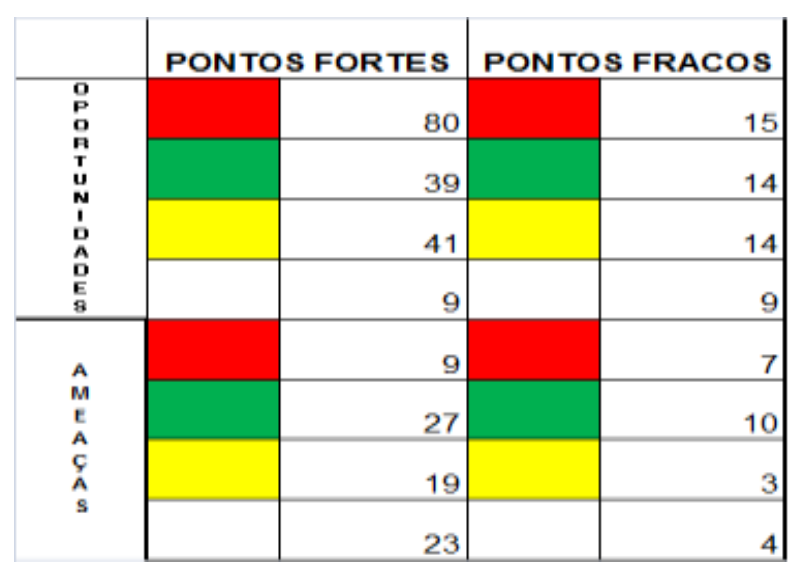

Figura 7 - Resumo da Postura Estratégica

$\mathrm{Na}$ postura estratégica de desenvolvimento, podem ser adotadas as seguintes estratégias: desenvolvimento do mercado atual ou abertura de novos; desenvolvimento de melhorias em produtos e ofertade serviços adicionais; desenvolvimento financeiro (por associações, busca de investidores); desenvolvimento de capacidades; desenvolvimento de estado de estabilidade (por associação ou fusão de empresas).

Após a análise ambiental, foram elaborados os seguintes objetivos estratégicos que ajudarão a empresa a definir onde empregar os recursos e os esforços a fim de a direcionaremquanto ao futuro desejado:

1 - Desenvolver indicadores de acompanhamento da saúde financeira a partir de janeiro de 2017;

2 - Ampliar programas de relacionamento com o cliente até julho de 2017;

3 - Aplicar pesquisa de satisfação de clientes a partir de janeiro de 2017, com periodicidade semestral;

4 - Implantar uma política de cobranças a partir de janeiro de 2017;

5 - Redefinir políticas de prazos até dezembro de 2017;

6 - Aperfeiçoar os colaboradores na área de vendas até agosto de 2017;

7 - Ampliar gradativamente investimentos em estratégias de marketing a partir de janeiro de 2018.

Os objetivos foram elaborados a partir da análise do ambiente externo e interno, que foi respondida pelo proprietário da empresa juntamente com as acadêmicas. Dentre as áreas analisadas no diagnóstico estratégico, estão a área financeira, marketing, tecnologia, recursos humanos e comercial, dessa forma, os objetivos estratégicos englobam todas as áreas da empresa.

$\mathrm{Na}$ definição dos objetivos estratégicos, todas as ações possuem prazos, para que essas sejam cumpridas até o período estabelecido ou iniciem nos prazos definidos, 
para que a empresa tenha um controle das ações que devem ser executadas prioritariamente, uma vez que o planejamento estratégico se dará nos próximos três anos, isto é até 2019.

E para que a empresa atinja de forma mais eficiente cada um dos objetivos estratégicos, foram elaborados planos de ações, onde nos mesmos são demonstrados os caminhos a serem seguidos para o alcance dos objetivos estratégicos.

Para ajudar a empresa no monitoramento do planejamento estratégico e dos planos de ação, pode-se fazer uso da ferramenta do BSC, onde o controle é feito a partir de indicadores de desempenho. $\mathrm{O}$ PDCA também pode ser proveitoso no que tange a implantação do planejamento, pois age de maneira com que os resultados possam ser controlados de forma que a eficiência de cada um possa ser ainda melhor, ou seja, trabalha-se com a melhoria continua.

No entanto, para facilitar o controle dos planos de ação da empresa em estudo, foi apresentada uma planilha de monitoramento e controle das ações estratégicas, de fácil entendimento e manuseio. A ferramenta tem em sua tela inicial, relacionados todos os objetivos estratégicos, ao clicar sob cada objetivo, este, redirecionará para outra planilha com as informações dos planos de ações, conforme figura 9 .

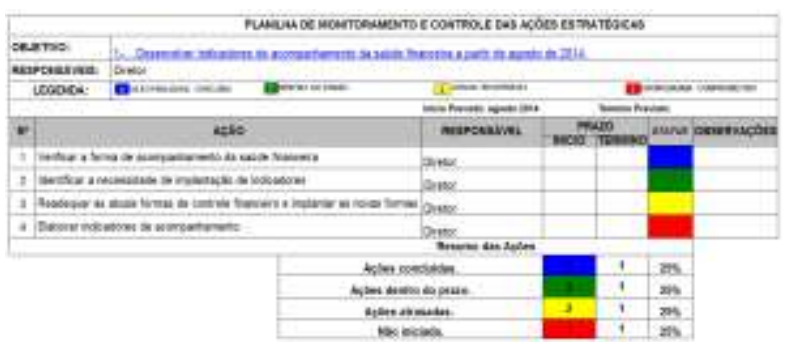

Figura 9 - Planilha de monitoramento e controle das ações estratégicas

Para o preenchimento da planilha, há uma coluna para identificar quem é o responsável pela execução das ações estratégicas bem como período de início e término. O status deve ser preenchido de acordo com o indicado na legenda e cada número corresponde a uma cor. Existe ainda uma coluna para eventuais observações a serem destacadas. Como uma informação importante para o gestor tem-se um resumo das ações, onde é apresentado o resultado do percentual de conclusão do objetivo estratégico analisado.

Para que seja possível a continuidade do controle dos demais objetivos estratégicos basta clicar sobre o próprio objetivo que retornará para a tela inicial da ferramenta.

Portanto, é uma ferramenta simples e de fácil preenchimento, porém de grande valor para a empresa, já que basta ao gestor ou responsável o correto preenchimento de todas as informações necessárias para o acompanhamento e o andamento de cada objetivo estratégico, bem como as suas ações estratégicas, verificando se o que foi planejado está sendo executado. Dependendo 
do status da ação, esta deverá ser revista e priorizada a fim de que o objetivo no todo se concretize.

\section{CONCLUSÃO}

O estudo buscou propor um planejamento estratégico, o qual foi construído e projetado para um período de três anos, sendo utilizado a partir desse momento como uma ferramenta para a gestão da empresa.

Foram desenvolvidos o negócio, missão, visão, valores e princípios da organização, tendo por base de sustentação os estudos realizados em teorias que apresentaram como elaborar esses propósitos.

Realizou-se a análise de ambiente, através da matriz SWOT, bem como a definição da postura estratégica, quando verificou-se que a empresa encontra-se no quadrante de desenvolvimento.

Definiram-se objetivos estratégicos para a empresa, para suas áreas afins e dessa forma elaborados objetivos estratégicos, bem como planos de ação para o alcance dos mesmos.

O estudo ainda apresentou e sugeriu uma ferramenta de monitoramento, avaliação e controle do planejamento estratégico

Destaca-se a relevância do planejamento estratégico para empresas deste ramo, pois a mesma não o possuía com essa estruturação, sendo que, se bem utilizado e revisto em um período determinado, será de grande valia e confiabilidade para a empresa, pois, o mesmo foi desenvolvido e elaborado em conjunto, empresário e acadêmicas.

\section{REFERÊNCIAS}

ANDRADE, Rosa de Andrade. Planejamento Estratégico: Formulação, Implantação e Controle. São Paulo; Atlas. ISBN; 978-85-2246799-0, 2012.

CHIAVENATO, Idalberto; SAPIRO, Arão. Planejamento Estratégico. Rio de Janeiro; Elsevier. ISBN; 85-352-1235-3, 2003.

COSTA, Eliezer Arantes da Gestão estratégica; da empresa que temos para a empresa que queremos. 2. Ed.-São Paulo; Saraiva. ISBN; 97885-02-06188-0, 2007.

KICH, Juliane Inês Francesco; PEREIRA, Mauricio Fernandes. Planejamento Estratégico: Os pressupostos básicos para uma implantação eficaz. Vol. 2; São Paulo, Atlas. ISBN; 978-85-2246187-5, 2011.

LOVATO, Adalberto. Metodologia da Pesquisa Três de Maio: SETREM. ISBN: 978-85-99020-050,2013

MATIAS-Pereira, José. Administração Estratégica: foco no planejamento estratégico. São Paulo; Atlas. ISBN: 978-85-224-6120-2, 2011.

MAXIMIANO, Antônio Cesar Amaru. Introdução à Administração. São Paulo. Atlas. ISBN: 85-2242164-1, 2000.

OLIVEIRA, Djalma de Pinho Rebouças de. Planejamento Estratégico: conceitos, metodologia e práticas. 31. Ed. - São Paulo: Atlas. ISBN: 97885-224-7485-1, 2013.

SAMPAIO, Cláudio Hoffmann. Planejamento Estratégico. 2.ed. - Porto Alegre: SEBRAE/RS, 1999.

VASCONCELOS FILHO, Paulo de; PAGNONCELLI. Dernizo. Construindo estratégias para competir no Século XXI. Rio de Janeiro; Elsevier. ISBN; 85-352-0767-8, 2001.

VIEIRA FILHO, Geraldo. Gestão da Qualidade Total: uma abordagem prática. 2. ed. São Paulo: Alínea, 2007.

13 